\title{
LA MUSIQUE
}

\section{DANS SA DIMENSION TEXTO-VISUELLE.} L'ANALYSE

DES CLIPS MUSICAUX NUMÉRIQUES

\author{
A MÚSICA NA SUA DIMENSÃO TEXTO-VISUAL. \\ ANALISE DE CLIPES MUSICAIS DIGITAIS \\ MUSIC IN ITS TEXTO-VISUAL DIMENSION. \\ DIGITAL MUSIC VIDEO ANALYSIS
}

Natalia Pimonova*

Université Sorbonne Paris Nord - Paris 13

\begin{abstract}
RÉSUMÉ: Cet article porte sur la dimension visuelle de la chanson commerciale, c’est-à-dire sur le clip musical, considéré comme l'ensemble synthétique de performances visuelle, textuelle et sociale (interactionnelle). Les approches théoriques sont l'analyse interactionnelle, l'analyse du discours et les popular music studies. La problématique de la recherche consiste en l'identification du rôle des clips vidéo dans l'iconisation du discours musical et s’ils peuvent être considérés comme des images mobiles numériques. Dans l'intention de répondre à cette problématique, nous visons à montrer comment les formes d'expression sémiotiquement hybrides iconisent le discours musical et comment la perception audiovisuelle de la musique est formée. L'étude porte sur l'analyse des clips vidéo des chansons sélectionnées et sur l'examen des moyens utilisés pour interagir avec le public: la corporéité et les gestes des artistes, les couleurs et les symboles. Le corpus est constitué des chansons officiellement certifiées pour leurs ventes importantes et listées par le site du SNEP (Syndicat National de l'Edition Phonographique).
\end{abstract}

MOTS CLÉS: Chanson commerciale. Popular music studies. Analyse du discours. Dimension texto-visuelle. Approche plurisémiotique.

RESUMO: Este artigo centra-se na dimensão visual da canção comercial, ou seja, o vídeo musical, considerado como o conjunto sintético de performances visuais, textuais e sociais (interaccionais). As abordagens teóricas são a análise interativa, a análise do discurso e os estudos musicais populares. O problema da investigação consiste em identificar o papel dos video clipes na iconização do discurso musical e se estes podem ser considerados como imagens em movimento digitais. A fim de abordar esta questão, pretendo mostrar como formas semi-híbridas de expressão iconizam o discurso musical e como a percepção audiovisual da música

* Doctorante à l'Université Sorbonne Paris Nord-Paris 13, ED Érasme (Pléiade). Directrice de recherche: Marie-Anne Paveau. E-mail:nataliapimonova@yahoo.com. 
é formada. Proponho, portanto, analisar os video clipes das canções selecionadas e distinguir os meios utilizados para interagir com o público: a corporeidade dos artistas e os seus gestos, cores e símbolos. O corpus consiste nas canções oficialmente certificadas para as suas importantes vendas e listadas pelo site do SNEP (Syndicat National de l'Edition Phonographique).

PALAVRAS-CHAVE: Popular music studies. Análise do discurso. Dimensão textual-visual. Análise interativa. abordagem multissemiótica.

ABSTRACT: This article examines visual dimension of a mainstream song, i.e., music video that is considered as a combination of visual, textual and social (interactional) performances. Main theoretical approaches are discourse analysis, interaction analysis and popular music studies. Human and social sciences often exclude music videos from their research data. The purpose of this study is to determine the role of music videos in iconisation of music discourse and to identify them as digital moving pictures. In order to answer these questions, the article considers music videos as a research data and contains an analysis of main methods of interaction with public, such as body language and gestures, colors and symbols. The research data consists of songs that were officially certified for their high sales by The National Syndicate of Phonographic Publishing (SNEP).

KEYWORDS: Mainstream song. Popular music studies. Discourse analysis. Texto-visual dimension. Plurisemiotic approach.

\section{INTRODUCTION}

Le développement des technologies a joué un rôle important dans la perception de la musique. La chanson est devenue une forme multimédiatique qui se regarde autant qu'elle s'écoute via les clips musicaux, principalement diffusés sur Internet ${ }^{1}$. Nous proposons donc d'étudier la dimension visuelle de la chanson, sans oublier sa dimension textuelle. En premier lieu, nous examinerons le clip musical, considéré comme un ensemble synthétique de performances visuelle, textuelle et sociale. En second lieu, nous nous pencherons sur le texte musical (ou les paroles de la chanson) dont nous étudierons les éléments lexicaux et sociaux.

La problématique principale de cet article est une marginalisation des clips musicaux sur le plan de la recherche en sciences humaines et sociales. Pourtant, la chanson commerciale occupe une place centrale sur le plan socio-économique (elle est au cœur de l'industrie musicale internationale). Pour répondre à cette problématique, nous tenterons de montrer comment le clip musical contribue au sens de la chanson et comment se forme la perception audiovisuelle de la musique.

La problématique secondaire consiste en l'identification du rôle des clips vidéo dans l'iconisation du discours musical. Sur le web, l'utilisation de l'image s'est considérablement développée et elle dépasse la simple illustration pour devenir une véritable porteuse de sens. En utilisant des formes d'expression sémiotiquement hybrides, l'iconisation du discours musical repose sur les paroles chantées (forme verbale) et sur la corporéité et les gestes des artistes, les couleurs et les symboles utilisés dans les clips musicaux (forme visuelle). L'iconisation peut être "définie à partir de la combinaison et de la coprésence du texte et de l'image" (PAVEAU, 2017, p. 307). Dans un même ordre d'idées, le clip musical peut être décrit comme une "nouvelle économie des signes sur l'espace de l'écran, [...] caractérisée par l'intégration de systèmes expressifs appartenant à plusieurs formats, image, texte et son, dans le même media" (AURAY, 2004, p. 97). Cette recherche comprend une analyse de toutes ces formes d'expression, mobilisées par les textes et les clips pour transmettre le message principal d'un titre musical. C'est par cette voie que l'énonciation musicale est devenue majoritairement visuelle, et le clip musical est devenu un moyen d'expression multimodal.

La question de recherche plus générale est celle de la construction de la représentation de la société française contemporaine dans les clips vidéo et les textes des chansons commerciales. La dimension textuelle de cette recherche est prise en charge par l'analyse de l'interaction et l'analyse du discours. Les domaines de la sociologie de culture et des popular music studies complètent l'analyse effectuée. Pour sa part, l'analyse visuelle a déjà été effectuée dans les articles suivants: Lebrun 2012; Gaudin 2018; Straw, 2018; Rossi, 2018; Boidy, 2018. Cette étude contribue donc à l'analyse du clip vidéo et propose une analyse du discours musical afin d'étudier la corrélation entre les dimension visuelle et textuelle de la chanson. Ces deux dimensions comprennent plusieurs éléments et créent l'image complète d'une chanson, afin de la transmettre au public telle quelle.

${ }^{1}$ Ce travail se base sur une recherche de thèse en cours, consacrée à l'analyse du discours de la chanson commerciale en France. 
Cet article se divise comme suit: la première partie explique le cadre théorique, la méthodologie et le choix du corpus; la deuxième partie est consacrée à l'analyse des phénomènes visuels et interactionnels; la conclusion apporte un regard critique sur les résultats et introduit quelques perspectives de cette recherche.

\section{CADRE THÉORIQUE ET MÉTHODOLOGIQUE}

La dimension visuelle tient actuellement une place prépondérante dans l'industrie musicale. D'un côté, le clip musical porte un caractère promotionnel, il est transmis à la télé et est accessible sur Internet, YouTube et d'autres plateformes digitales basées sur les informations en forme de vidéo. Généralement, "le vidéo clip constitue un objet central dans le vaste univers audiovisuel contemporain" (GAUDIN, 2018, p. 97). De l'autre côté, le rôle des textes musicaux et de la musique elle-même ne peut pas être diminué, ces deux éléments constituant la base de la chanson. Will Straw affirme d'ailleurs que "au-delà de YouTube, le clip vidéo joue désormais un rôle assez secondaire dans les scénarios possibles concernant le futur (et la renaissance) de l'industrie musicale" (STRAW, 2018, p. 187).

Cette recherche s'est surtout concentrée sur l'analyse de l'image mobile numérique qui devient un acteur important de l'industrie musicale contemporaine. Le clip vidéo est diffusé en ligne afin d'être accessible et disponible pour un grand public. Il devient une sorte "d'image conversationnelle, produit inattendu de la rencontre de la numérisation des contenus visuels et de l'interaction documentée" (GUNTHERT, 2014, p. 1). Nous proposons donc d'analyser principalement les clips vidéo des chansons sélectionnées et de distinguer les moyens utilisés pour interagir avec le public: la métaphore visuelle, les signes et les symboles, les couleurs et le langage corporel. Le message de la chanson commerciale contemporaine est souvent transmis à travers la vidéo et ses formes d'expression plurisémiotiques.

Dans ce contexte, l'approche méthodologique choisie est qualitative. Le but est de faire l'analyse multimodale de l'interaction, des clips vidéo et du rapport image-texte, et enfin de montrer comment la parole n'existe pas sans le corps et comment la chanson influence les perceptions sensorielles par écran. Selon la sociolinguistique et l'analyse du discours numérique, l'interaction a un caractère multimodal: elle doit donc faire l'objet d'une analyse langagière, mais aussi d'une analyse des images, des sons et d'autres outils graphiques et visuels de communication. Christine Develotte et Marie-Anne Paveau décrivent ainsi la multimodalité de la chanson telle qu'elle est présentée sur Internet: "Il s'agit d'espaces multimodaux faisant co-exister des éléments graphiques, iconiques et picturaux, statiques ou mobiles, et des fonctions de communication synchrones et asynchrones" (DEVELOTTE et PAVEAU, 2017, p. 206).

En raison de la dématérialisation de la musique, l'évaluation des ventes et du succès d'un titre musical est calculée par les ventes digitales et non physiques. C’est pourquoi le format Internet des clips musicaux est privilégié dans cet article. Afin de constituer le corpus de travail, les outils numériques suivants ont été utilisés:

- $\quad$ le site des certifications musicales - SNEP (Syndicat National de l'Edition Phonographique) pour sélectionner des titres musicaux ayant les meilleures ventes (cela constitue la garantie que ces chansons sont au sommet des productions musicales contemporaines). Disponible en: http://www.snepmusique.com/les-disques-diamant. Accès 06 jui. 2021.

- la plateforme digitale YouTube pour collecter les clips musicaux des chansons sélectionnées. Disponible en: https://www.youtube.com/watch. Accès 06 jui. 2021.

- le site Paroles2chansons pour recueillir la version texte des chansons analysées. Disponible en: https://paroles2chansons.lemonde.fr/. Accès 06 jui. 2021.

- le site Genius pour recueillir la version texte des chansons analysées. Disponible en: https://genius.com/. Accès: 06 jui. 2021. 
Grâce à leur statut mondial, des chansons anglophones récentes (sur les cinq dernières années) ont été choisies pour faire l'analyse de l'image et évaluer sa contribution au sens de la chanson. Le choix des années 2017 - 2019 est en effet le plus pratique pour constituer un corpus actuel. La liste des chansons choisies pour l'analyse figure dans l'annexe 1.

Les premier et deuxième critères de sélection sont donc la date de sortie récente et le succès d'un titre musical. Le troisième critère est le contenu de la chanson qui peut potentiellement concerner une problématique sociale et être intéressant pour l'analyse textovisuelle. Enfin, un certain impact social/politique et l'effet produit sur le web (parfois les titres musicaux deviennent même des hymnes politiques non officiels) forment le quatrième critère.

Nous présentons maintenant les résultats de recherche sur Google, également appelés SERP (Search Engine Results Page, soit: page de résultats du moteur de recherche). Ces résultats énumèrent les pages web et les sources où les chansons sélectionnées ont été mentionnées. Chaque titre a constitué un phénomène de société par: la publication d'articles, l'enregistrement de reprises (des covers) ou de vidéos avec l'explication des clips musicaux ou des messages portés par le texte de telle ou telle chanson. Les chiffres indiqués ci-dessous montrent que ces titres musicaux sont populaires et font l'objet de très nombreuses recherches sur Internet:

- $\quad$ Katy Perry ft. Skip Marley, "Chained to the rhythm" (PERRY, 2017): 540000 résultats sur Google;

- $\quad$ Childish Gambino, "This is America" (GLOVER, 2018): 5580000 résultats sur Google;

- $\quad$ Beyoncé ft. Blue Ivy, Saint JHN, WizKid, "Brown Skin Girl" (BEYONCÉ, 2020): 494000 résultats sur Google.

\section{LES TRAVAUX D'ANALYSE TEXTO-VISUELLE}

Comme il a été précisé plus haut, la transmission du sens de la chanson et l'interaction artiste-public sont possibles grâce à la coexistence de la parole et du corps. À cet effet, nous proposons d'inclure le discours dans l'analyse de la dimension visuelle de la chanson. L'approche multimodale implique en effet que le discours comprend plusieurs phénomènes et que les termes discours et interaction sont mutuellement dépendants et connectés. Catherine Kerbrat-Orecchioni explique d'ailleurs que le terme de "discours en interaction" remplace aujourd'hui celui "d'interactions" (KERBRAT-ORECCHIONI 2006, p. 138). Le croisement des moyens d'expression visuels et lexicaux conditionne donc l'existence de pratique visio-discursive:

[...] l'interaction s'organise donc non seulement sur la base, fondamentale, de l'organisation verbale des tours de parole, mais aussi à travers l'exploitation située des ressources multimodales, à travers les gestes, la disposition des corps dans l'espace ainsi que le recours à des artefacts variés. (KERBRAT-ORECCHIONI, 2005, p. 131-132)

La première chanson analysée est "Chained to the rhythm" de Katy Perry. Le sens de cette chanson est exprimé conjointement par les paroles et le clip vidéo. Le clip est réalisé dans le style des années $1950^{2}$ avec quelques influences et détails futuristes. L'action se passe dans le parc "Oblivia" (l'oubli, la rêvasserie). Grâce aux vêtements, aux couleurs et aux manèges présents dans le clip, on réalise que la vie créée pour les citoyens était et restera imaginaire et même dystopique. La chanson critique le système existant et appelle à élaborer une nouvelle vision: "'Chained to the Rhythm' est d'ailleurs un hymne au lâcher prise et à la liberté dans une société de plus en plus étouffante" (GONCALVES, 2017, page web).

Le message socio-politique est dominant dans cette composition. L'artiste invoque une époque très particulière dans l'histoire étatsunienne: pendant les années 1950, les gens ont été vraiment emportés par l'idée de l'American Dream (le rêve américain) et "enchaînés" au rythme de vie commun. Voici quelques sous-thématiques abordées par la chanson de Katy Perry et représentées aux niveaux visuel et lexical:

\footnotetext{
${ }^{2}$ Les années 1950 couvrent la période de 1950 à 1959. Dans cet article, on évoque surtout les traits de la vie sociale et culturelle des États-Unis qui ont été caractéristiques pour cette période.
} 
- $\quad$ La répétition. La vie de la société est prise dans une boucle, tout se répète et on est obligé de suivre ce rythme établi. Au niveau visuel, on voit une roue de hamsters (voir Figure 1) utilisée par les gens qui font la queue afin de participer à cette course infinie. Plusieurs répétitions et l'utilisation excessive des anaphores soutiennent l'idée d’une vie monotone et répétitive:

\begin{tabular}{|c|c|}
\hline Texte original & Traduction Française \\
\hline $\begin{array}{c}\text { "... So comfortable, we're livin' in a bubble, bubble } \\
\frac{\text { So comfortable, we cannot see the trouble, trouble }}{<\ldots>} \\
\text { Come on, turn it up, keep it on repeat..." } \\
\text { (extrait de Katy Perry ft. Skip Marley "Chained to the rhythm", } \\
\text { lignes } 5-6,18 \text { ) }\end{array}$ & $\begin{array}{c}\text { "...C'est si confortable, on vit dans une bulle, une bulle } \\
\text { C'est si confortable, ces problèmes qu'on occulte, qu'on occulte } \\
<\ldots> \\
\text { Allez, monte le son, passe-le en boucle..." }\end{array}$ \\
\hline
\end{tabular}

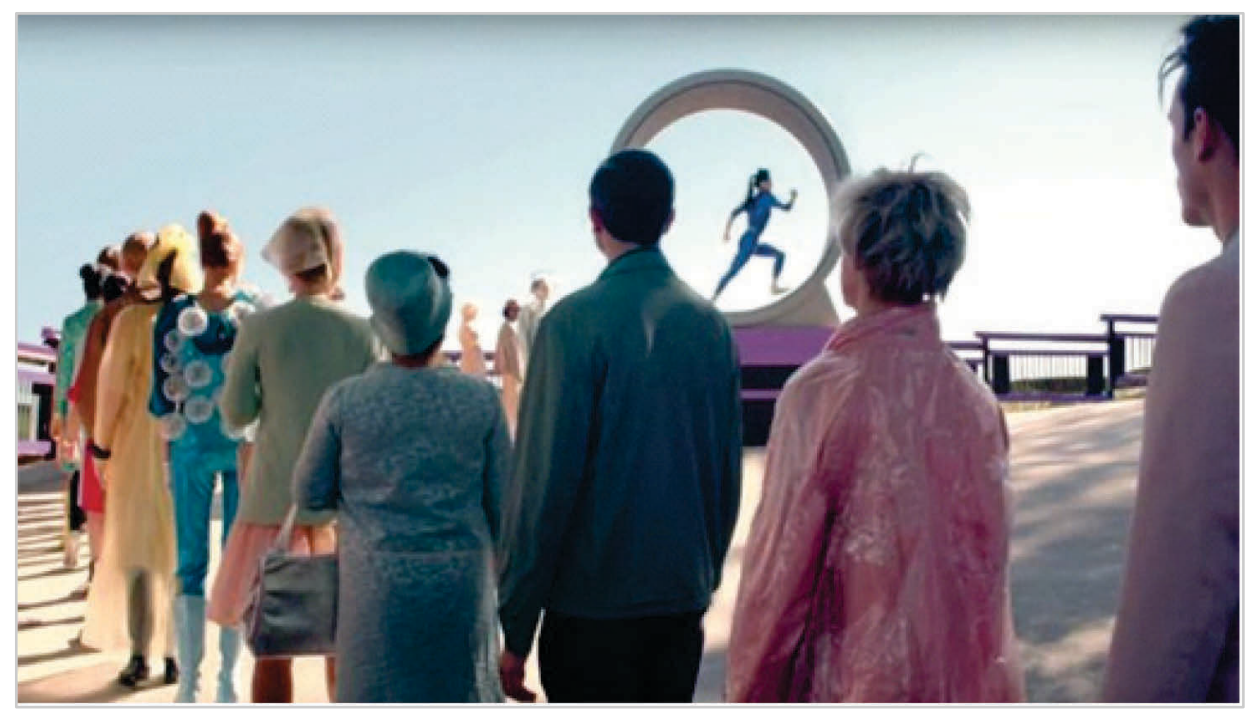

Figure 1: Capture d'écran du clip musical "Chained to the rhythm" par Katy Perry

Source: Perry (2017)

- $\quad$ Lillusion. Notre vision du monde est illusoire et des problèmes réels sont toujours cachés cette façade. Ce message est exprimé aux niveaux visuel (la présence des couleurs irréelles et des attractions qui nous rappellent des vrais problèmes de société) et textuel:

\begin{tabular}{c|c} 
Texte original & Traduction Française \\
\hline "...Are we crazy? & "Sommes-nous fous? \\
Living our lives through a lens & Nopped in our white-picket fence \\
Like ornaments..." & Nous vivons nos vies à travers un objectif \\
Enfermés derrière nos barrières aux piquets blancs
\end{tabular}

- $\quad$ La dépendance aux écrans. La vidéo suggère une addiction nuisible aux réseaux sociaux, au cinéma et à la vie sans aucun engagement social ou politique, exprimée au niveau du lexique et de l'image. Dans la vidéo, on voit les gens qui regardent exclusivement les écrans de leurs téléphones portables ou l'écran du cinéma. Tous les spectateurs sont au cinéma en portant les lunettes 3D, et nous pouvons faire l'hypothèse que cela signale une image unique imposée par les autorités (voir Figure 2). Lors de 
cette séance de cinéma, la seule personne qui enlève ses lunettes pour voir au-delà de l'écran est la chanteuse (voir Figure 3). Les paroles portent également sur le problème de la vie trompeusement insouciante:

\begin{tabular}{c|c} 
Texte original & Traduction Française \\
\hline ...So put your rose-colored glasses on & \\
And party on & \\
$<\ldots>$ & "... Alors, vois la vie en rose \\
Stumbling around like a wasted zombie..." & Et continue à faire la fête \\
& $<\ldots>$
\end{tabular}

(extrait de Katy Perry ft. Skip Marley "Chained to the rhythm",

lignes $14-15$ et 19)

Tu titubes et trébuches, mort vivant, complètement soul..."

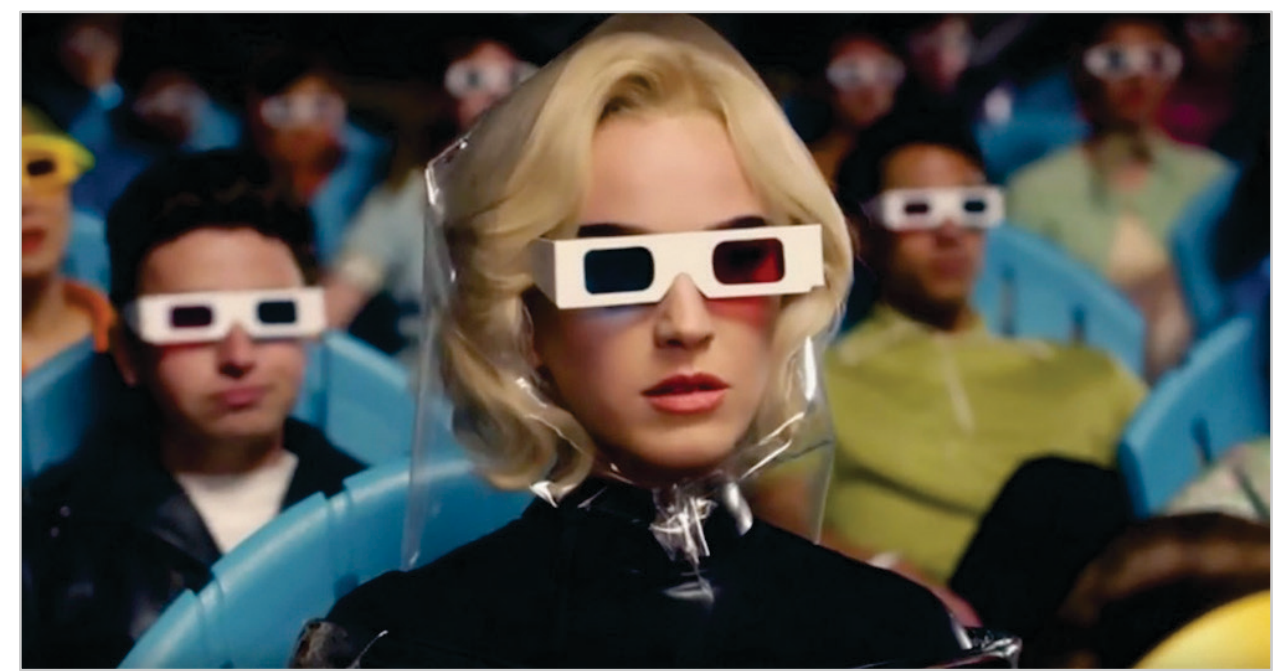

Figure 2: Capture d'écran du clip musical "Chained to the rhythm" par Katy Perry

Source: Perry (2017)

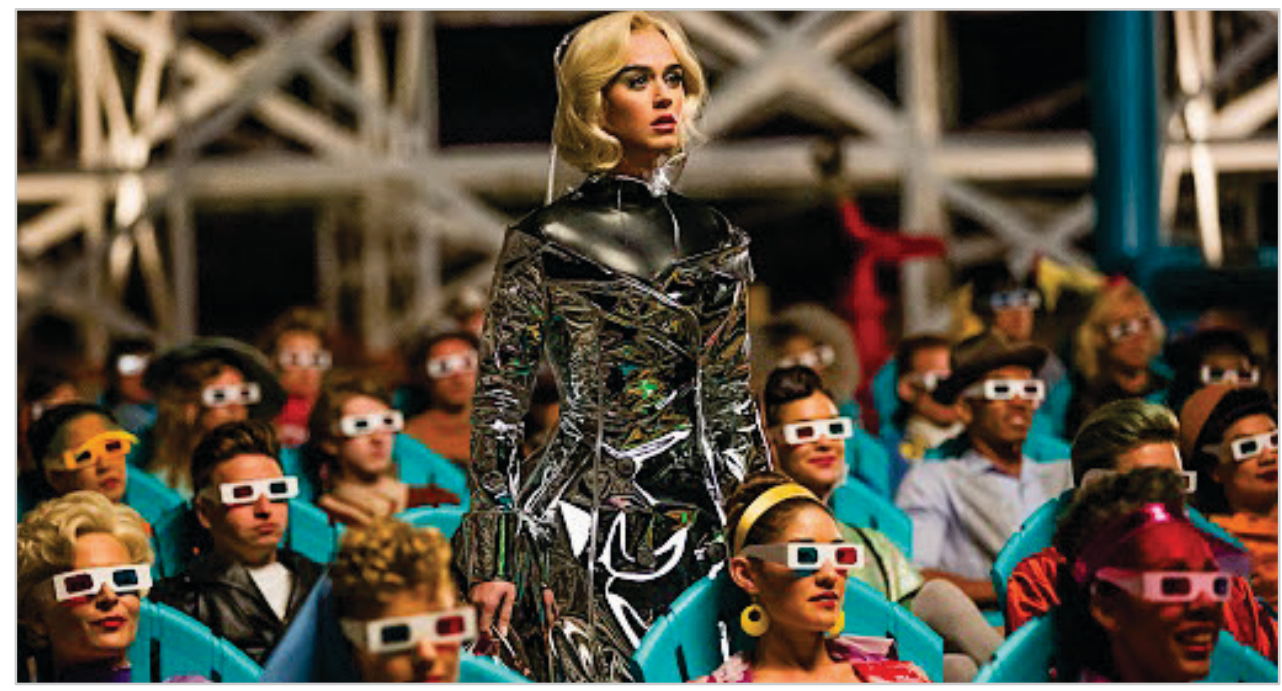

Figure 3. Capture d'écran du clip musical "Chained to the rhythm" par Katy Perry

Source: Perry (2017)

On peut donc dire que ce titre de Katy Perry soulève plusieurs questions problématiques pour la société contemporaine, qui se formulent à travers des phénomènes visuels, lexicaux et poétiques. 
Le deuxième clip vidéo analysé se concentre sur le langage du corps. L'artiste Childish Gambino utilise ce moyen d'expression afin de transmettre le message de la chanson "This is America". Les mimiques et la gestuelle sont des outils d'interaction efficaces et usuels, qui servent à construire la communication entre l'artiste et le public, comme le souligne Lorenza Mondada: "L'interaction s'organise grâce à une pluralité de ressources multimodales: les gestes, les regards, les postures corporelles, les mouvements, l'agencement spatial des participants" (MONDADA, 2005, p. 111)

La chanson de Childish Gambino est consacrée aux violences et injustices (notamment les violences de la police contre les personnes noires aux États-Unis). Des processus et des phénomènes sociaux sont souvent reflétés dans les productions musicales "sans jamais perdre de vue la structure sociale hiérarchique et les inégalités matérielles qui en découlent" (VUILLE et al., 2009, p. 4). La violence des événements décrits dans la chanson "This is America" est montrée aux niveaux textuel et visuel puisque le chanteur et ses danseur.euse.s la montrent à l'aide de la danse et des expressions faciales. Elles sont prononcées, et même exagérées, cherchant à représenter la perception raciste ${ }^{3}$ des personnes noires. Dans la figure 4 , on peut remarquer entre autres la coiffure du chanteur qui est élaborée dans le style afro, ainsi que la reprise d'une figure publicitaire raciste, les deux soutenant l'idée d'une perception raciste des noir.e.s.
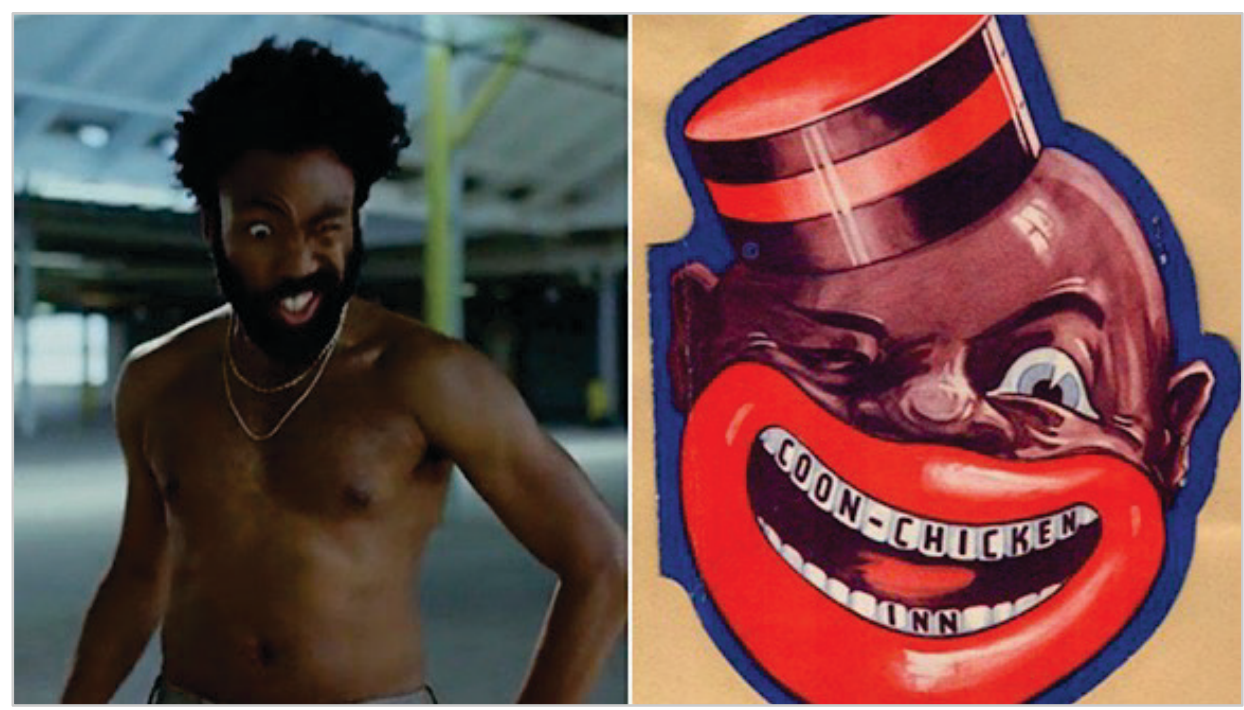

Figure 4: Capture d'écran du clip musical "This is America" par Childish Gambino

Source: Glover (2018)

Au niveau textuel, il y a dans cette chanson des passages entiers consacrés aux diverses violences et à l'omniprésence des armes dans les quartiers noirs:

\begin{tabular}{c|c} 
Texte original & Traduction Française \\
\hline "...Look at how I'm livin' now \\
Police be trippin' now (woo) \\
Yeah, this is America (woo, ayy) \\
Guns in my area (word, my area) \\
\hline Igot the strap (ayy, ayy) \\
Igotta carry'em..." \\
La police est en train de commettre des bavures (woo) \\
Ouais, ici, c'est l'Amérique (woo, ayy) \\
Des flingues dans mon quartier (mon quartier) \\
J'ai la bandoulière (ayy, ayy)
\end{tabular}

L'idée que notre vision du monde est illusoire se manifeste également dans cette chanson. Les problèmes réels restent cachés derrière l'envie de vivre sa vie sans engagement social ou politique:

\footnotetext{
${ }^{3}$ Aux XIX-XX siècles aux États-Unis, les capacités physico-artistiques et les expressions faciales des personnes noires étaient ridiculisées et caricaturées. C’est un des traits caractéristiques de la perception et de l'image racistes.
} 


\begin{tabular}{c|c} 
Texte original & Traduction Française \\
\hline ...We just wanna party \\
$<\ldots>$ \\
I know you wanna party \\
Party just for me \\
Girl, you got me dancin' (yeah, girl, you got me dancin') \\
Dance and shake the frame..." \\
$\begin{array}{c}<\ldots \text {... } \\
\text { (extrait de Childish Gambino "This is America", lignes } 1,5-8)\end{array}$
\end{tabular}

En outre, l'artiste Childish Gambino parle du destin d'un homme noir dans la société étatsunienne, des violences policières contre les personnes noires. On voit la peur du chanteur exprimée par ses mimiques: il tente d'échapper aux agents de police (voir Figure 5). Le thème de l'inégalité des races devant la loi et la police est abordé:

\begin{tabular}{|c|c|}
\hline Texte original & Traduction Française \\
\hline 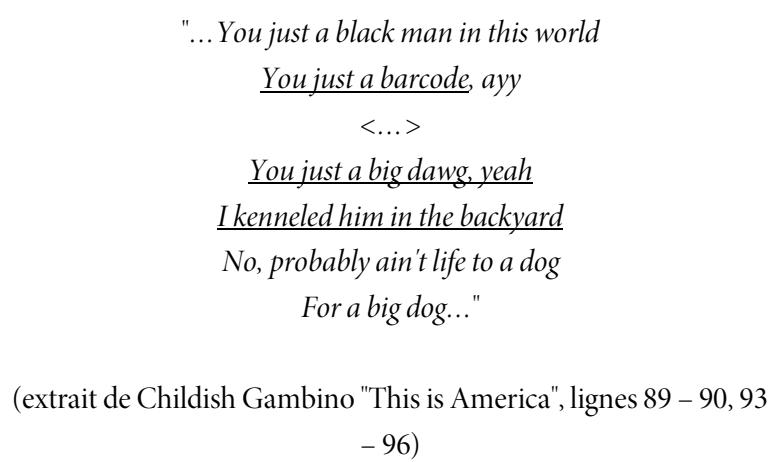 & $\begin{array}{c}\text { "...T'es juste un Noir dans ce monde } \\
\text { T'es juste un code barre, ayy } \\
<\ldots> \\
\text { T'es juste un gros chien, yeah } \\
\text { Je l'ai mis en cage dans le jardin } \\
\text { Ce n'est probablement pas une vie pour un chien } \\
\text { Pour un gros chien..." }\end{array}$ \\
\hline
\end{tabular}

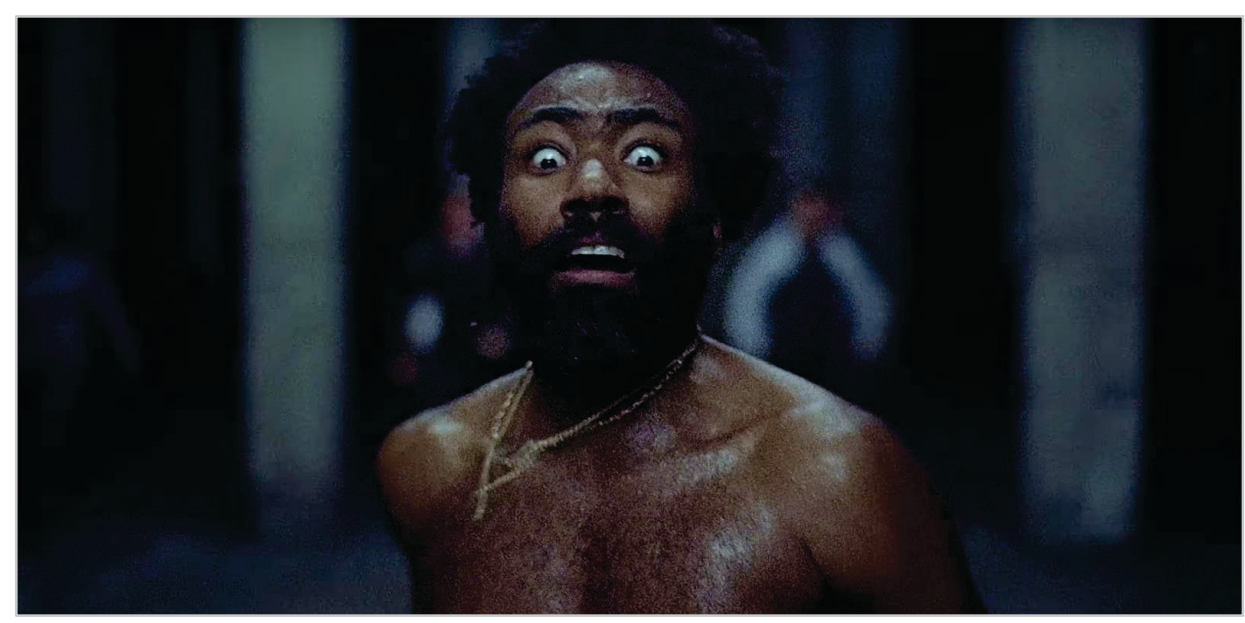

Figure 5: Capture d'écran du clip musical "This is America" par Childish Gambino Source: Glover (2018)

Pour conclure l'analyse de la chanson "This is America", nous voudrions citer Myriam Haegel, l'auteure qui offre quelques explications complémentaires de ce titre musical: 
Pour comprendre cette hallucination musicale, il faut remonter le cours du temps [...] Childish Gambino en Jim Crow ${ }^{4}$, symbole raciste, abattant de sang-froid un homme noir déshumanisé par une cagoule [...] Certain.es estiment qu'en dansant devant des horreurs, Childish Gambino dénonce le fait que la société met en avant le divertissement, pour détourner l'attention des sujets graves (HAEGEL, 2019, page web).

La dernière chanson étudiée dans cet article est consacrée aux femmes noires, à leurs beauté, peau, coiffures et force. Le clip vidéo "Brown Skin Girl" réunit les femmes noires et métisses ayant des âges, des religions et des parcours différents. L'interaction se réalise à travers le lexique (les paroles de la chanson, comme dans l'exemple suivant, qui contient un discours valorisant sur la "peau brune") et la corporéité (les cadres du clip): cela forme une pratique discursive multimodale.

\begin{tabular}{|c|c|}
\hline Texte original & Traduction Française \\
\hline $\begin{array}{c}\text { "...Brown skin girl } \\
\text { Your skin just like pearls } \\
\text { The best thing in the world } \\
\text { Never trade you for anybody else..." } \\
\text { (extrait de Beyoncé ft. Blue Ivy, Saint JHN, WizKid "Brown Skin } \\
\text { Girl", lignes } 1-4 \text { ) }\end{array}$ & $\begin{array}{c}\text { "...Fille à la peau brune } \\
\text { Ta peau est comme des perles } \\
\text { La meilleure chose dans le monde } \\
\text { Je ne te changerai pour personne d'autre..." }\end{array}$ \\
\hline
\end{tabular}

Le clip musical devient une production artistique construite "sur l'emploi d'images importées associées à des séquences musicalisées, ce qui indiquerait un fonctionnement particulier, propre à ce que l'on pourrait appeler la discursivisation de la musique" (PEQUIGNOT, 2018, p. 122). Dans l'intention de transmettre le message principal de la chanson et de rendre hommage à la culture et au physique des personnes noires, les artistes introduisent des cadrages qui mettent en valeur les coupes de cheveux des femmes noires et la diversité de leurs formes (voir Figure 6). Par ailleurs, Beyoncé, Blue Ivy, Saint JHN et WizKid sont soutenus par d’autres personnes noires connues ${ }^{5}$ aux États-Unis. Elles se réunissent dans le clip vidéo "Brown Skin Girl" (voir Figure 7).

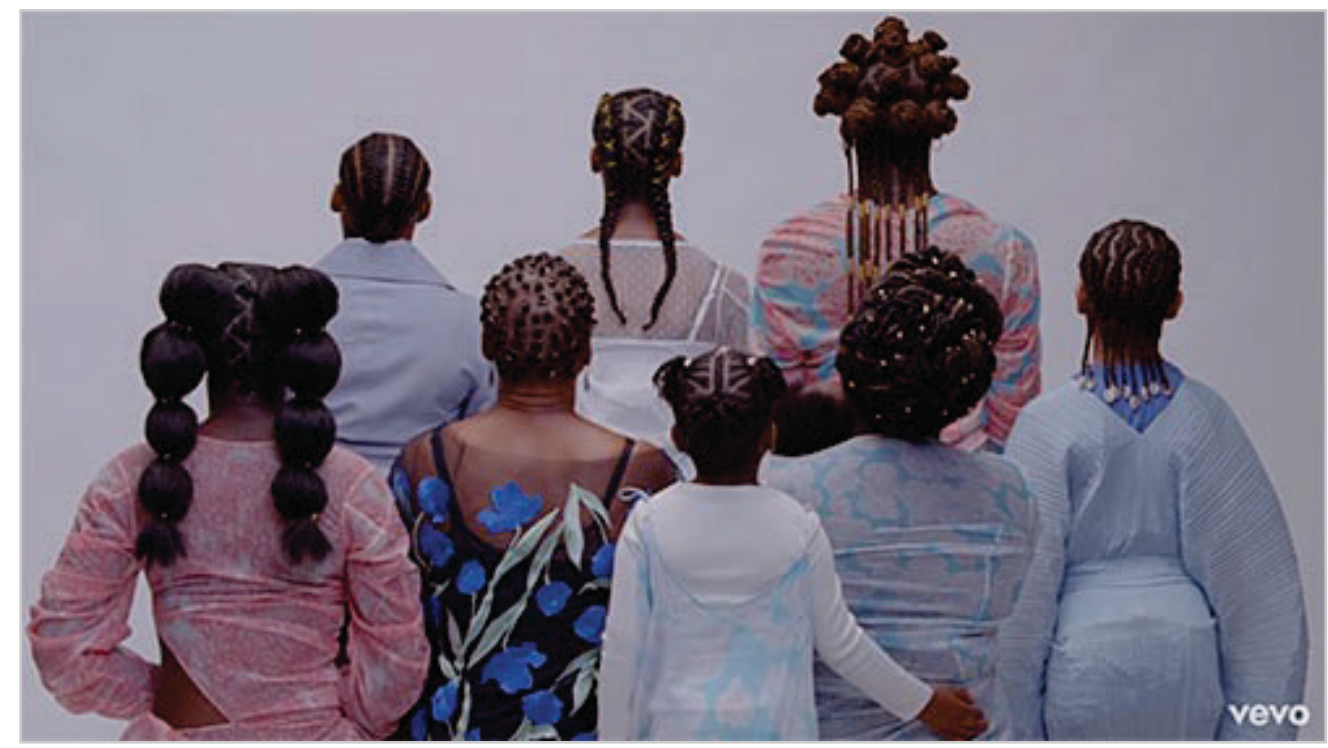

Figure 6: Capture d'écran du clip musical "Brown Skin Girl" par Beyoncé ft. Blue Ivy, Saint JHN, WizKid

Source: Beyoncé (2020)

\footnotetext{
${ }^{4}$ Jim Crow - est un personnage incarnant un esclave d'origine africaine et popularisé par une chanson "Jump Jim Crow" (1828); pendant des décennies Jim Crow a été le symbole de la ségrégation et du racisme anti-noir. A présent, il apparaît comme le symbole du mouvement antiraciste.

${ }^{5}$ La présence des collègues, des amis et des membres de famille des artistes. Par exemple, on voit la chanteuse américaine Kelly Rowland et la fille aînée de Beyoncé, Blue Ivy (voir Figure 8). En outre, Naomi Campbell (la mannequin britannique, d'ascendance jamaïcaine) et Lupita Nyong'o (l’actrice et réalisatrice mexico-kényane) font leur apparition dans le clip vidéo.
} 


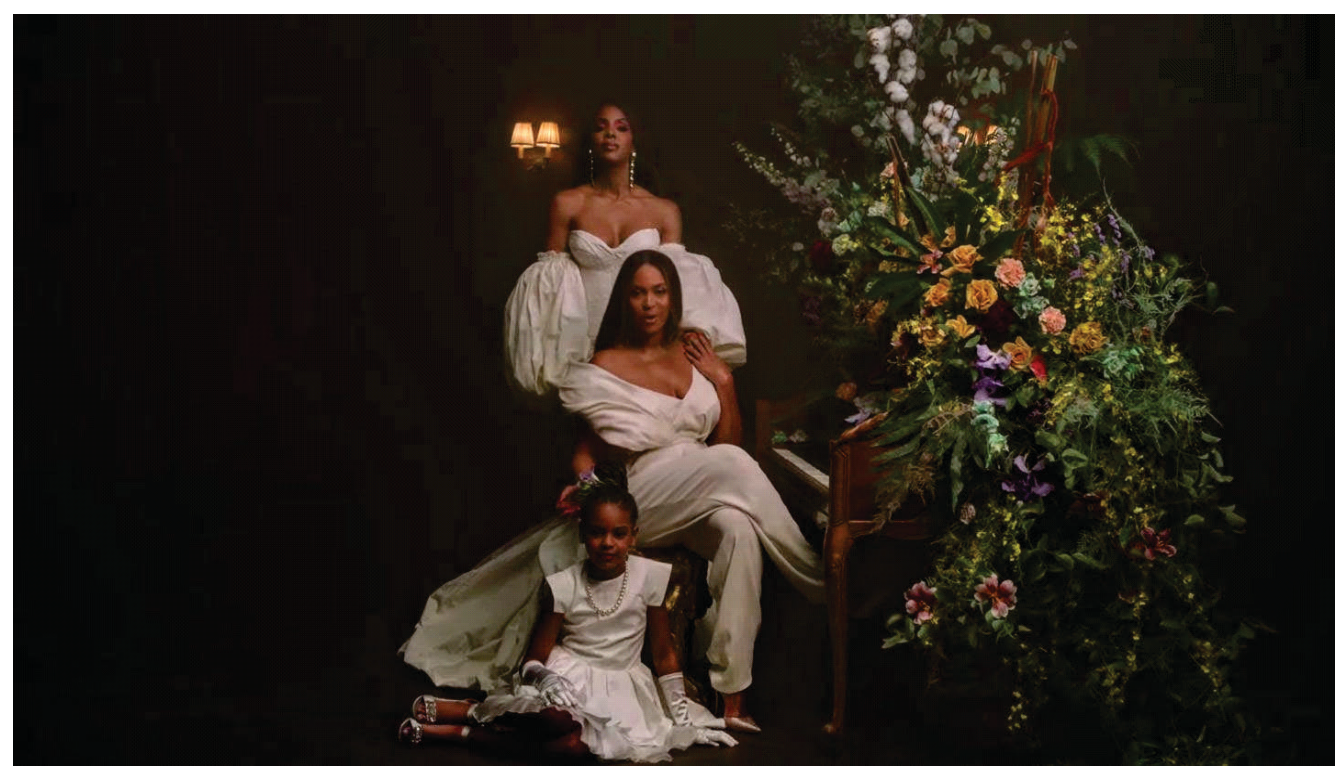

Figure 7: Capture d’écran du clip musical "Brown Skin Girl" par Beyoncéft. Blue Ivy, Saint JHN, WizKid

Source: Beyoncé (2020)

Parallèlement aux moyens visuels utilisés dans le clip musical de Beyoncé, Blue Ivy, Saint JHN et WizKid, les célébrités étatsuniennes et la beauté de la peau noire sont également mentionnées au niveau textuel:

\begin{tabular}{|c|c|}
\hline Texte original & Traduction Française \\
\hline $\begin{array}{l}\text { "...Pose like a trophy when } \underline{\underline{\text { Naomi's }}} \text { walk in } \\
\text { She need an Oscar for that pretty dark skin } \\
\text { Pretty like Lupita when the cameras close in } \\
\text { Drip broke the levy when my killas roll in } \\
\text { I think tonight she might braid her braids } \\
\text { Melanin too dark to throw her shade } \\
\text { She minds her business and winds her waist } \\
\text { Gold like } 24 k \text {, okay..." } \\
\text { (extrait de Beyoncé ft. Blue Ivy, Saint JHN, WizKid "Brown Skin Girl", } \\
\text { lignes } 27-34 \text { ) }\end{array}$ & $\begin{array}{l}\text { "... Tu poses comme un trophée quand entre Naomi } \\
\text { Il faut un oscar pour cette jolie peau sombre } \\
\text { Jolie comme Lupita quand se rapprochent les caméras } \\
\text { Mon style a tout cassé quand mes Kellys sont arrivées } \\
\text { Je crois que ce soir, elle pourrait faire ses tresses } \\
\text { Sa mélanine est trop sombre pour me faire de l'ombre } \\
\text { Elle s'occupe de ses affaires et ondule de la taille } \\
\text { C'est de l'or, genre vingt-quatre carats, d'accord..." }\end{array}$ \\
\hline
\end{tabular}

La chanson en version audio est sortie en 2019, mais la véritable reconnaissance et la popularité sont arrivées avec la sortie du clip vidéo officiel en 2020. Cela confirme que le clip musical est un instrument puissant utilisé pour enrichir et promouvoir la chanson. Les plateformes numériques de distribution musicale contribuent donc à la promotion des titres et des clips musicaux, y étant euxmêmes enrichis par les outils numériques, comme le souligne Jamie Sexton: "Les technologies digitales rendent les clips musicaux plus importants" (SEXTON, 2010, p. 99 [notre traduction]). 


\section{CONCLUSION}

Depuis quelques décennies, la musique populaire se manifeste dans une dimension visuelle: il est donc logique d'inclure des clips musicaux dans l'étude de la chanson et de montrer l'investissement des moyens d'expression visuels dans les interactions effectuées à travers le clip musical. Étant donné que la chanson est actuellement accessible sur Internet, il est possible de la traiter en ces deux dimensions, textuelle et visuelle. À ce propos, Fabrice Bousteau indique:

Ce qui m’anime depuis des années, c'est justement la relation des images et des mots [...]. Toute image est un texte et toute image a pour origine un texte. À la conception de toute image, il y a son projet, formulé par des mots. Et à la suite de toute image, il y a possibilité de la décrire, de la commenter. (BOUSTEAU, 2009, p. 122, 125)

Au fil des années, sont parus beaucoup d'articles et d'ouvrages analysant la chanson commerciale et sa contribution à la vie sociale, culturelle et politique (Calvet, 1981; LeBlanc, Boudreault-Fournier et Djerrahian, 2007; Hammou, 2008; Gaudin, 2018 etc.). En même temps, il existe peu de recherches qui parlent du discours musical, par exemple Bonnet, 2013; Sylvanise, 2015; Lesacher, 2016; Péquignot, 2018. Ces études sont assez récentes et elles ne proposent pas de véritable analyse du discours. C’est pourquoi prendre en compte la dimension visuelle de la chanson commerciale constitue une innovation pour l'analyse du discours et l'analyse de l'interaction.

La centralité du clip musical reste une question contradictoire: est-ce le texte ou l'image qui prime? Cela dépend de la sélection du corpus, certains titres musicaux se focalisant sur l'image, ou sur le texte, ou sur les deux.

D’une part, c'est la dimension textuelle, donc langagière, qui reste dominante dans la chanson populaire. Parmi les titres musicaux qui ont été analysés dans le travail réalisé pour la thèse, certains, comme la chanson "Mauvaise graine" de Nekfeu voient leur sens construit par des paroles de chanson, sans contribution significative du clip vidéo. Si les éléments textuels dominent dans la chanson commerciale, on peut la percevoir comme un texte relativement court, poétique et portant un message complet. Dans ce cas, le rôle de la musique et de l'image devient secondaire dans la construction du sens de la chanson. Nous estimons cependant que cette perception est partiale parce qu'elle exclut les divers éléments de la chanson qui en font une composition multimodale.

D’autre part, l'importance des textes des chansons a diminué pendant le siècle des nouvelles technologies et des plateformes digitales, et le centre d'attention est actuellement sur l'image et sur le clip vidéo. Selon les résultats d'analyse effectuée dans cet article, l’image prime ou joue un rôle équivalent au texte pour construire le sens de la chanson populaire: les moyens d'expression visuels et sonores sont souvent utilisés pour interagir avec le public et le clip musical devient un outil d'analyse autosuffisant. À travers des couleurs, des vêtements, des gestes ou des mimiques utilisés dans les vidéos, il est possible de construire un rapport image-texte.

La chanson commerciale est "un objet souvent considéré comme vulgaire par les universitaires" (BONNET, 2013, p. 175). Nous espérons que cette recherche modifie cette position et confirme la place de la chanson populaire parmi les objets de la recherche universitaire. Il est vrai que la musique commerciale contient souvent un lexique et des images qu'on peut considérer comme vulgaires, ou non standards, mais en même temps, il s’agit de moyens efficaces pour faire passer le message souhaité au public.

La perception de la chanson est devenue multimodale, car le développement des clips vidéo, des ventes et des téléchargements réalisés en ligne crée plus de possibilités de création et de consommation musicales. Cela rend un titre musical multifacette. C’est pourquoi il était important de mettre en valeur l'analyse du clip musical dans ce travail. Selon les exemples présentés ci-dessus, le clip musical peut être porteur de sens au même niveau que les paroles de la chanson (comme on l'a montré en Perry, 2017; Glover, 2018 et Beyoncé, 2020) ou même devenir un outil d’analyse autosuffisant (la vidéo "This is America" de Childish Gambino). Nous en concluons que la contribution de la dimension visuelle à la mise en valeur du texte de la chanson commerciale contemporaine est considérable. Ce résultat est partiel (valable pour cet article et son corpus), en même temps, il constitue un indicateur pour l'analyse de la musique populaire de manière plus générale.

\section{RÉFÉRENCES}


AURAY, N. Sosies et avatars dans les jeux: entre écriture et image. L'image Sosie. L'original et son double. In: Actes du $1^{\text {er }}$ colloque international ICONE-IMAGE. Sens, Musées de Sens. 8-10 juillet, 2004, p. 95-108.

BEYONCÉ. Blue Ivy, SAINt JHN, WizKid - BROWN SKIN GIRL (Official Video). Youtube. 24 août 2020. Disponible en: https://www.youtube.com/watch?v=vRFS0MYTC1I. Accès: 06 jui. 2021.

BOIDY, M. Corps, gestes, interpellations. Iconographie politique du format clip. Volume! v. 14, n. 2, 2018, p. 175-186. Disponible en: https://journals.openedition.org/volume/5581. Accès: 15 avr. 2021.

BONNET, G. La Chanson populittéraire. Texte, musique et performance. Paris: Kimé, 2013, p. 175-176.

BOUSTEAU, F. Bruno Racine et Alain Fleischer font de la lecture un spectacle. magazine Beaux Arts, n 305, 1 novembre 2009, p. $122-125$.

CALVET, L.-J. Chanson et société. Langages et Sociétés. Paris: Payot, 1981.

DEVelotTe, C., PAVEAU, M.-A. Pratiques discursives et interactionnelles en contexte numérique. Questionnements linguistiques. Langage et société, n. 160-161, p. 199-215, 2017. Disponible en: https://www.cairn.info/revue-langage-et-societe2017-2-page-199.htm. Accès: 25 oct. 2020.

GAUDIN, A. Le clip comme forme d'expression musico-visuelle: pour une esthétique de la relation musique-images. Volume! 2018 , p. 97-110. Disponible en: http://journals.openedition.org/volume/5556. Accès: 29 oct. 2020.

GLOVER, D. Childish Gambino - This Is America (Official Video). Youtube. 06 mai. 2018. Disponible en: https://www.youtube.com/watch?v=VYOjWnS4cMY. Accès: 06 jui. 2021.

GONCALVES, J. "Chained to the Rhythm": Katy Perry délire et s'engage dans un clip politique. Purebreak charts. 2017. Disponible en: http://www.chartsinfrance.net/Katy-Perry/news-103728.html - aqcU6ZSRmYyAq5d7.99. Accès: 11 mai. 2021.

GUNTHERT, A. L'image conversationnelle. Études photographiques, n.31, Printemps 2014. Disponible en: http://journals.openedition.org/etudesphotographiques/3387. Accès: 12 mai. 2021.

HAEGEL, M. Avec This is America, Childish Gambino remporte 3 prix aux Grammy Awards 2019. Madmoizelle, 11 février 2019. Disponible en: http://www.madmoizelle.com/this-is-america-childish-gambino-917561. Accès: 1 mai. 2021.

HAMMOU, K. La vérité au risque de la violence. Remarques sur la stylistique du rap en français. In: MOÏSE, C., AUGER, N.; SCHULTZ-ROMAIN, C. FRACCIOLLA, B. De l'impolitesse à la violence verbale. Avignon: L'Harmattan, 2008.

KERBRAT-ORECCHIONI, C. Le discours en interaction. Paris: Armand Colin, 2005.

KERBRAT-ORECCHIONI, C. Comptes rendus. Revue française de linguistique appliquée, v. XI, n.2, p. 138-144, 2006. Disponible en: https://www.cairn.info/revue-francaise-de-linguistique-appliquee-2006-2-page-138.htm. Accès: 11 mai. 2021.

LEBLANC, M.-N.; BOUDREAULT-FOURNIER, A.; DJERRAHIAN, G. Les jeunes et la marginalisation à Montréal: La culture hip-hop francophone et les enjeux de l'intégration. Diversité urbaine, Montréal, v.1, n .7, p. 9-29, 2007.

LEBRUN, B. Chanson et performance: mise en scène du corps dans la chanson française et francophone. Paris: L'Harmattan, 2012. 
LESACHER, C. Rap, genre, langage et québéquicité: enjeux et tensions sociolinguistiques de l'accès aux espaces médiatiques à Montréal. Cahiers internationaux de sociolinguistique, v. 10, n. 2, p. 233-256, 2016.

MONDADA, L. Chercheurs en interaction. Comment émergent les savoirs. Lausanne: Presses Polytechniques et Universitaires Romandes, 2005.

PAVEAU, M.-A. L'analyse du discours numérique. Dictionnaire des formes et des pratiques. Paris: Hermann Editeurs, 2017.

PERRY, K. Chained To The Rhythm (Official) ft. Skip Marley. 21 fev. 2017. Youtube. Disponible en: https://www.youtube.com/watch?v=Um7pMggPnug. Accès: 06 jui. 2021.

PEQUIGNOT, J. Clip et discours: pragmatique de l'énonciation. Volume! v. 14, n. 2, p. 111-124, 2018. Disponible en: https://journals.openedition.org/volume/5572. Accès: 28 oct. 2020.

ROSSI, J. "L'homme au bouquet de fleurs" de Maxime Le Forestier: le clip comme approfondissement de la chanson. Volume!, v. 14, n. 2, p. 125-136, 2018.. Disponible en: https://journals.openedition.org/volume/5575. Accès: 18 avr. 2021.

SEXTON, J. Digital music: production, distribution and consumption. In: CREEBER, G.; MARTIN, R. (ed.). Digital cultures: understanding new media. Maidenhead: Open University Press, 2010. p. 92-106.

STRAW, W. Le clip vidéo et ses contextes: 30 ans plus tard. Volume! v. 14, n. 2, 2018. Disponible en: https://journals.openedition.org/volume/5589. Accès: 27 oct. 2020.

SYLVANISE, F. À la recherche d'une poétique ou comment lire une chanson populaire américaine. In: GHERMANI, L.; MURILLO, C.; TORTI-ALCAYAGA. Tisseurs: transtextualisation et interculturalités. Itinéraires. Literature, texte, culture, 2014-2, 2015. Disponible en: https://journals.openedition.org/itineraires/2486. Accès: 15 mai. 2021.

VUILLE, M., MALBOIS, F., ROUX, P., MESSANT, F. et PANNATIER, G. Comprendre le genre pour mieux le défaire. Nouvelles questions féministes, v. 28, n. 3, p. 4-14, 2009.

\section{() (1) $\circledast$}

Reçu le 23 février 2021. Accepté le 20 mai 2021. 